\title{
Article
}

\section{The Beijing Twin Study (BeTwiSt): An Update}

\author{
Dandan $\mathrm{Bi}^{1,2}$, Xinying $\mathrm{Li}^{1,2}$, Jie Chen ${ }^{1}$, Nengzhi Jiang ${ }^{1,2}$ and Jianxin Zhang ${ }^{1}$ \\ ${ }^{1}$ CAS Key Laboratory of Mental Health, Institute of Psychology, Chinese Academy of Sciences, Beijing, China and ${ }^{2}$ Department of Psychology, University of \\ Chinese Academy of Sciences, Beijing, China
}

\begin{abstract}
The Beijing Twin Study (BeTwiSt), which was established in 2006, is an ongoing study aiming to investigate the genetic and environmental etiology of adolescent psychopathology. Resting-state brain imaging datasets have been examined for same-sex twins, and other psychological traits and emotional and behavioral variables have been examined for all twins. Based on the registry, the main findings regarding the etiological mechanism underlying adolescent development, magnetic resonance imaging results, and genetic and environmental influences on other psychological traits have been published. This article summarizes the key findings in these three areas and discusses future plans for the BeTwiSt.
\end{abstract}

Keywords: Twin study; adolescent; psychopathology; magnetic resonance imaging; psychological traits

(Received 17 May 2019; accepted 18 July 2019; First Published online 29 August 2019)

\section{History of the Beijing Twin Study}

The Beijing Twin Study (BeTwiSt) is a longitudinal twin project established in 2006 to investigate the genetic and environmental etiologies of emotional and behavioral problems in children and adolescents in China (Chen, Li, Zhang et al., 2012). This study offers a unique opportunity to understand the effects of the genes and environmental factors and their interplay on the development of psychopathology within a longitudinal framework. The variables of interest in the study include demographic characteristics (e.g., age, sex and ethnic group), biological factors (e.g., puberty, height and weight), adolescent psychopathology (e.g., depression, anxiety and problematic behavior), psychological processes (e.g., emotion regulation, rumination and effortful control) and social variables (e.g., life stress, parenting and peer affiliation). Psychopathological variables for parents (e.g., depression, anxiety, drinking and smoking) were also explored. Emotional and behavioral information was provided for the twins by multiple informants, including the twins, their parents and their teachers, to resolve rater bias in the assessment. In addition, saliva samples were provided by the twins to extract their genomic DNA using the Oragene DNA self-collection kit (Genotek Inc.).

The zygosity of same-sex twins in the study was determined by a combination of DNA analyses and a questionnaire (Chen, Li, Chen et al., 2012). Among 1125 pairs of same-sex twins (T1 sample), 990 pairs were determined by genetic analyses, and 116 pairs, whose saliva samples yielded insufficient DNA, were determined by the questionnaire method. Zygosity of 19 pairs was unable to be identified because of missing responses to the zygosity questionnaire and insufficient DNA. For the genetic analyses, DNA was purified from saliva samples and used to amplify the following nine short

Author for correspondence: Xinying Li, Email: lixy@psych.ac.cn

Cite this article: Bi D, Li X, Chen J, Jiang N, and Zhang J. (2019) The Beijing Twin Study (BeTwiSt): An Update. Twin Research and Human Genetics 22: 486-491, https://doi.org/ 10.1017/thg.2019.52 tandem repeat loci: D3S1358, D13S317, D8s1179, vWA, D16S539, D18S51, D5S818, D21S11 and FGA. All of these nine loci, which are highly heterogeneous in the Chinese population, were used in the genetic analysis. Same-sex twins with at least one different genetic marker were categorized as dizygotic (DZ) twins, whereas other same-sex twins were categorized as monozygotic (MZ) twins. The zygosity of twins whose saliva samples yielded insufficient DNA was identified via a questionnaire. The predictive accuracy of the questionnaire method in this study was estimated at $91 \%$ when compared with genotyping (Chen, Li, Chen et al., 2012). The zygosity of a small number of twins was determined by questionnaire, but these may have been misclassified because of the $91 \%$ accuracy. According to the simulations study by Neale (2003), there are biased estimates of variance components using conventional analysis for samples containing misclassified pairs, showing that the additive genetic variance (A) is underestimated while shared environmental variance $(\mathrm{C})$ and no-shared environmental variance $(\mathrm{E})$ are overestimated.

\section{Advances of the BeTwiSt}

\section{Completion of Three Waves of Data Collection}

Participants were recruited based on a large, representative twin sample from over 600 public schools in Beijing, China. At present, the study has completed three waves of data collection: T0 for a pilot survey, T1 for a comprehensive survey and T2 for a follow-up survey. The intervals between waves were approximately 1.5 years in duration. The core samples in the BeTwiSt were T1 and T2, which consisted of 1387 pairs of twins and 1007 pairs of twins, respectively. The attrition rate from $\mathrm{T} 1$ to $\mathrm{T} 2$ was $27.4 \%$. Descriptions of the samples in the T1 and T2 waves, including zygosity, sex and age, are shown in Table 1 . The power of this study to detect the effects of common environmental variation was calculated by Mx (http://www.vcu.edu/mx). The analysis result 
Table 1. Description of samples in waves 1 and 2

\begin{tabular}{|c|c|c|c|c|c|}
\hline \multirow[b]{2}{*}{ Zygosity } & \multirow[b]{2}{*}{ Sex } & \multicolumn{2}{|c|}{ T1 wave } & \multicolumn{2}{|c|}{ T2 wave } \\
\hline & & $n$ (pairs) & Mean age $(S D)$ & $n$ (pairs) & Mean age $(S D)$ \\
\hline \multirow{3}{*}{$M Z$} & Male & 380 & $13.94(2.54)$ & 279 & $15.34(2.53)$ \\
\hline & Female & 415 & $14.23(2.84)$ & 331 & $15.71(2.82)$ \\
\hline & Sum & 795 & $14.09(2.70)$ & 610 & $15.54(2.70)$ \\
\hline \multirow[t]{3}{*}{ DZ } & Male & 146 & $13.63(2.73)$ & 98 & $15.12(2.83)$ \\
\hline & Female & 165 & $14.22(2.77)$ & 124 & $15.57(2.72)$ \\
\hline & Sum & 311 & $13.94(2.77)$ & 222 & $15.37(2.78)$ \\
\hline OST & & 262 & $13.63(2.62)$ & 169 & $15.16(2.49)$ \\
\hline \multirow[t]{3}{*}{ Unknown zygosity } & Male & 9 & $15.42(1.97)$ & 3 & $14.78(1.09)$ \\
\hline & Female & 8 & $14.99(3.12)$ & 3 & $17.29(2.73)$ \\
\hline & Sum & 19 & $15.22(2.54)$ & 6 & $16.03(2.38)$ \\
\hline Total & & 1387 & $13.99(2.71)$ & 1,007 & $15.44(2.69)$ \\
\hline
\end{tabular}

Note: $M Z=$ monozygotic twins, $D Z=$ dizygotic twins, OST = opposite-sex twins.

showed that based on a combined observed sample size of 1368 (T1 wave, $\mathrm{MZ}=795, \mathrm{DZ}=573$ ), at the .05 significance level with $1 d f$, power of this study was 0.9680 when $A=20 \%, 0.9999$ when $\mathrm{A}=40 \%, 1.0000$ when $\mathrm{A}=60 \%, 0.2978$ when $\mathrm{C}=10 \%, 0.9944$ when $\mathrm{C}=30 \%$ and 1.0000 when $\mathrm{C}=50 \%$. These results were in line with expectations that powers are high except when $\mathrm{C}=10 \%$.

\section{Resting-State Functional Magnetic Resonance Imaging Scanning}

The effects of both genetic and environmental factors can be mediated by brain structure and function (Jansen et al., 2015). Evidence shows that resting-state brain activities play an important role in the etiology of child and adolescent psychopathology (Menon, 2011). Therefore, we collected resting-state functional magnetic resonance imaging (fMRI) datasets from 108 pairs of same-sex twins after the T2 wave to determine how the resting-state brain activities were influenced by genes and the environment in adolescence and whether this brain activity mediated the relationship between genetic and environmental risk factors and behavioral outcomes. These participants, including 27 male and 27 female MZ twin pairs and 29 male and 25 female DZ twin pairs, were recruited via telephone invitation. The twins' mean age was $15.72(S D=0.94)$ years.

After reading and signing the informed consent form, the scanning procedure was initiated. Images were acquired with a $3.0 \mathrm{~T}$ Siemens Trio Tim scanner. A three-dimensional T1-weighted volumetric sequence was performed using a protocol with repetition time $=2530 \mathrm{~ms}$, echo time $=3.37 \mathrm{~ms}$, flip angle $=7^{\circ}$, field of view $=256 \times 256 \mathrm{~mm}^{2}$, acquisition matrix $=256 \times 192 \mathrm{~mm}^{2}$, slice thickness $=1.33 \mathrm{~mm}$ without gap and slice number $=144$. Restingstate fMRI data were obtained via an echo-planar imaging sequence with the following parameters: echo time $=30 \mathrm{~ms}$, repetition time $=2 \mathrm{~s}$, flip angle $=90^{\circ}$, acquisition matrix $=64 \times 64 \mathrm{~mm}^{2}$, field of view $=220 \times 220 \mathrm{~mm}^{2}$, slice thickness $=4 \mathrm{~mm}$ without gap and 180 volumes. The scanning time was $6 \mathrm{~min}, 30 \mathrm{~s}$. Conventional functional imaging preprocessing was performed using Statistical Parametric Mapping (SPM12, https://www.fil.ion.ucl.ac.uk/spm/ software/spm12/). The brain imaging data were merged with behavioral and genetic datasets, and data analysis is ongoing.

\section{Measurement of Other Psychological Traits}

Considering that various complex human traits are influenced by genes and environment (Polderman et al., 2015), we collaborated with researchers in other fields to examine the genetic base of individual differences across various domains in adolescents. Data collection included social and personality (e.g., narcissism, liking for names, life satisfaction, affective wellbeing, impulsive buying, gender-science stereotypes, nostalgia, self-enhancement, implicit self-esteem and mistrust), cognitive [e.g., biological motion (BM) perception, spatial working memory and set-shifting] and other (e.g., autistic traits and risk-taking) factors. Table 2 provides the measurement methods for the specific phenotypes in which we were interested.

\section{Key Findings of the BeTwiSt}

\section{The Etiological Mechanism Underlying Adolescent Externalizing and Internalizing Problems}

ACE models have been used to estimate the contributions of genetic and environmental factors to adolescent psychopathology. Regarding internalizing problems, ACE models were established for depression and anxiety symptoms (Table 3). Moderate heritability was found for both depression and anxiety in Chinese adolescents, and non-shared environmental factors played an important role (Chen, Li, Natsuaki et al., 2014; Chen, Yu, Li et al., 2015; Chen, Yu, Zhang et al., 2014). These results were generally consistent with previous findings observed in Western adolescents, supporting the crosscultural generalizability of the etiological models of adolescent depression and anxiety. Concerning externalizing problems, ACE models were established for problematic internet use, aggression and rule-breaking behavior (Chen, Yu, Liu et al., 2015; Li et al., 2014). The results also revealed a moderate genetic influence on these externalizing problems. Non-shared and shared environmental effects were also observed, although the latter was generally small. As rumination and dysfunctional attitudes are often correlated with depression (Krause et al., 2017; Winkeljohn Black \& Pössel, 2015), we further estimated the genetic and environmental sources of these two variables and observed moderate heritability in Chinese adolescents for both, which suggests that rumination and dysfunctional 
Table 2. Measurement methods for the specific phenotypes in the BeTwiSt

\begin{tabular}{|c|c|c|}
\hline Phenotype & & Measurement method \\
\hline Narcissism & & $\begin{array}{l}\text { Questionnaire (the Narcissistic Personality } \\
\text { Inventory, the Communal Narcissism Inventory, } \\
\text { the Narcissistic Grandiosity Scale, the Psychological } \\
\text { Entitlement Scale) }\end{array}$ \\
\hline Liking for names & & One item \\
\hline Life satisfaction & & Questionnaire (the Satisfaction with Life Scale) \\
\hline Affective wellbeing & & Questionnaire (the Scale of Positive and Negative Experience) \\
\hline Impulsive buying & & Questionnaire (the Impulsive Buying Scale) \\
\hline \multirow{2}{*}{$\begin{array}{l}\text { Gender-science } \\
\text { stereotypes }\end{array}$} & Explicit & Questionnaire (two 7-point Likert-type scales) \\
\hline & Implicit & Implicit Association Test \\
\hline Nostalgia & & Questionnaire (the Southampton Nostalgia Scale) \\
\hline Self-enhancement & & Better-Than-Average Task \\
\hline Implicit self-esteem & & Implicit Association Test \\
\hline Mistrust & & Questionnaire (the Social Mistrust Scale) \\
\hline Biological motion perception & & Intact and Scrambled Biological Motion Sequences \\
\hline Spatial working memory & & Spatial Working Memory Task \\
\hline Set-shifting & & Intra-Extra Dimensional Set-Shifting task \\
\hline Autistic traits & & Questionnaire (the Autism-Spectrum Quotient) \\
\hline Risk-taking & & Balloon Analogue Risk Task \\
\hline
\end{tabular}

Table 3. Genetic and environmental influences (\%) on emotional and behavioral problems in adolescents in the BeTwiSt

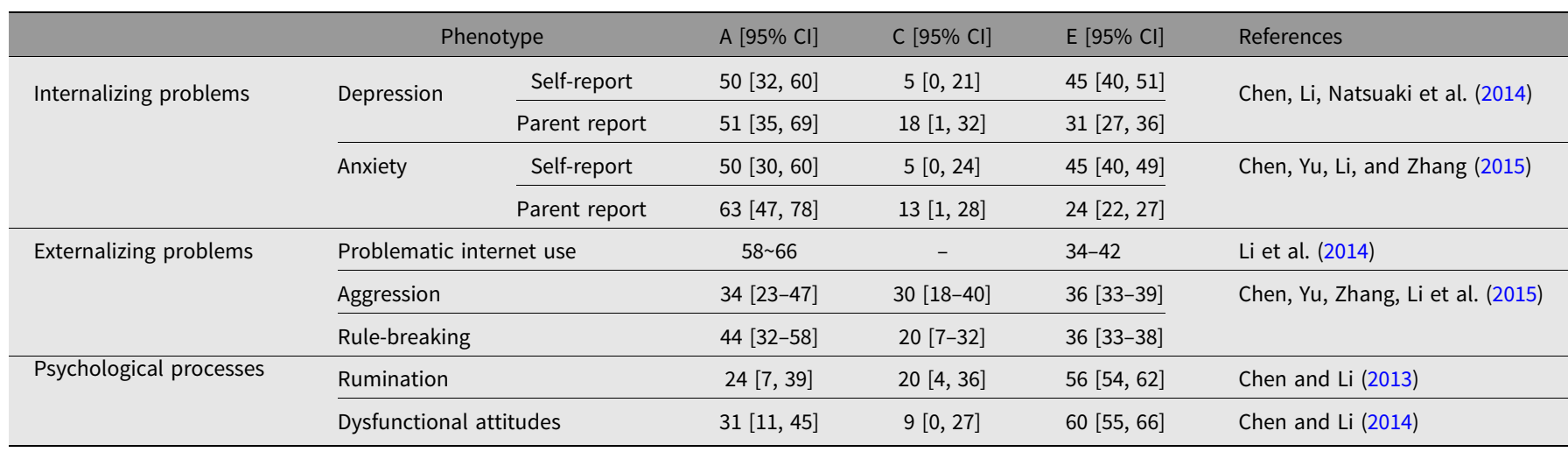

Note: $\mathrm{A}=$ additive genetic effects, $\mathrm{C}=$ shared environmental effects, $\mathrm{E}=$ no-shared environmental effects.

attitudes can be used as an endophenotype reflecting genetic risk of depression (Chen \& Li, 2013, 2014).

Consensus that gene-environment $(\mathrm{G} \times \mathrm{E})$ interactions contribute to the development of adolescent depression and anxiety has been reached. However, knowledge about specific genetic and environmental factors involved in $\mathrm{G} \times \mathrm{E}$ interactions is limited. Therefore, we conducted molecular behavioral genetic studies to investigate the interaction effect between specific genes and environmental factors on the etiology of adolescent depression and anxiety. Given that the BDNF plays an important role in the growth and differentiation of neurons and may be sensitive to changes in environment (Park \& Poo, 2012), the BDNF gene is a promising candidate for $\mathrm{G} \times \mathrm{E}$ interactions. We genotyped $B D N F$ Val66Met polymorphism and tested the interaction between this polymorphism and some specific environmental factors (e.g., maternal parenting and stressful life events) on adolescent depression and anxiety. Results showed that the BDNF Val66Met polymorphism significantly moderated the influence of maternal positive parenting and stressful life events on adolescent depression and anxiety (Chen, Li, \& McGue, 2012, 2013; Chen, Yu, Liu et al., 2015; Zhang et al., 2016). These findings provided evidence to support the differential susceptibility hypothesis of youth depression and anxiety, which suggests that individuals with sensitive genes are more susceptible to both positive and negative environmental influences, while less sensitive individuals are less susceptible to changes in the environment.

As non-shared environment has been found to exert a significant effect on both internalizing and externalizing problems (Table 3), we used the MZ difference design to investigate the effect of specific non-shared environmental factors on adolescent psychopathology. The basic assumption of MZ difference design is that all the observed behavioral differences between $\mathrm{MZ}$ twin pairs are due to non-shared environments, as the two twins have 
Table 4. Heritability estimates for the social and personalistic factors in the BeTwiSt

\begin{tabular}{|c|c|c|c|c|c|c|}
\hline \multirow[b]{2}{*}{ Phenotype } & & \multicolumn{2}{|c|}{$\begin{array}{l}\text { Number of } \\
\text { twin pairs }\end{array}$} & \multirow[b]{2}{*}{ Best-fitting model } & \multirow[b]{2}{*}{ Heritability $[95 \% \mathrm{CI}]$} & \multirow[b]{2}{*}{ References } \\
\hline & & $M Z$ & $\mathrm{DZ}$ & & & \\
\hline \multirow{2}{*}{ Narcissism } & Communal narcissism & \multirow{2}{*}{152} & \multirow{2}{*}{152} & $\mathrm{AE}$ & $0.42[.29, .53]$ & \multirow{2}{*}{ Luo, Cai, Sedikides et al. (2014 } \\
\hline & Agentic narcissism & & & $\mathrm{AE}$ & $0.47[.36, .57]$ & \\
\hline \multirow[t]{2}{*}{ Narcissism } & Intrapersonal narcissism & 152 & 152 & $\mathrm{AE}$ & $0.42[.30, .53]$ & \multirow[t]{2}{*}{ Luo, Cai, and Song (2014) } \\
\hline & Interpersonal narcissism & & & $\mathrm{AE}$ & $0.35[.21, .47]$ & \\
\hline \multicolumn{2}{|l|}{ Liking for names } & 152 & 152 & $\mathrm{AE}$ & $0.47[.36, .57]$ & \multirow[t]{3}{*}{ Luo, Shi et al. (2014) } \\
\hline Life satisfaction & & & & $\mathrm{AE}$ & $0.45[.33, .55]$ & \\
\hline Affective wellbeing & & & & $\mathrm{AE}$ & $0.40[.27, .51]$ & \\
\hline \multirow[t]{2}{*}{ Narcissism } & Maladaptive narcissism & 152 & 152 & $\mathrm{AE}$ & $0.44[.31, .55]$ & \multirow[t]{3}{*}{ Cai et al. (2015) } \\
\hline & Adaptive narcissism & & & $\mathrm{AE}$ & $0.37[.24, .49]$ & \\
\hline Impulsive buying & & & & $\mathrm{AE}$ & $0.34[.20, .47]$ & \\
\hline \multirow[t]{2}{*}{ Gender-science stereotypes } & Implicit & 152 & 152 & AEU & $0.38(.30, .46)$ & \multirow[t]{2}{*}{ Cai et al. (2016) } \\
\hline & Explicit & & & AEU & $0.18[.08, .29]$ & \\
\hline Nostalgia & & 117 & 115 & $\mathrm{AE}$ & $0.30[.14, .45]$ & Luo et al. (2016) \\
\hline Self-enhancement & & & & $\mathrm{AE}$ & $0.37[.20, .51]$ & \\
\hline Implicit self-esteem & & 117 & 115 & $\mathrm{AE}$ & $0.36[.22, .49]$ & Cai and Luo (2017) \\
\hline \multirow[t]{2}{*}{ Mistrust } & Home mistrust & 616 & 140 & ACE & $0.40[.18, .66]$ & \multirow[t]{2}{*}{ Zhou, Wong et al. (2018) } \\
\hline & School mistrust & & & ACE & $0.26[.03, .54]$ & \\
\hline
\end{tabular}

Note: $\mathrm{A}=$ additive genetic effects, $\mathrm{C}=$ shared environmental effects, $\mathrm{E}=$ no-shared environmental effects, $\mathrm{U}=$ error/unreliability.

almost the same genes. One study showed that there were different related patterns between parental warmth reasoning and harsh hostility in youth anxiety, indicating that parental warmth reasoning and harsh hostility may exert unique environmental influences on youth anxiety (Chen et al., 2016). Another study found that the relationship between adolescent externalizing behaviors and parental hostility supported the selection effect, in which the twin with a higher level of externalizing behaviors was more likely to be subject to greater hostility relative to his or her co-twin; this was also supported by the relationship between externalizing behaviors and deviant peer affiliation (Hou et al., 2013).

\section{Findings of Magnetic Resonance Imaging}

There is an advantage to integrating behavior genetics and magnetic resonance imaging (MRI) to investigate the heritability of brain morphology and function. Researchers have shown that brain activation in cortical motor areas, the thalamus and the cerebellum was associated with complex motor sequencing and audiovisual integration ( $\mathrm{Li}$, Huang et al., 2018), and activation in the left insula, right stratum and right superior parietal lobule was risk-related and may be heritable (Rao et al., 2018). In addition, researchers scanned the resting state of adolescent twins and found large genetic correlations between head motion and the default network intrinsic functional connectivity, which had profound implications for interpreting individual differences in default network connectivity (Zhou et al., 2016). They also found that the subdivisions of diverse brain regions based on genetic correlations were generally consistent with functional connectivity patterns, indicating that the magnitude of the genetic covariance in brain anatomy could be used to portray the boundaries of functional subregions of the brain (Cui et al., 2016).

\section{Genetic and Environmental Influences on Social and Personality Factors}

The BeTwiSt has produced numerous important results regarding the genetic and environmental bases of some vital social and personality factors such as different categories of narcissism (Table 4; Cai \& Luo, 2017; Cai et al., 2016; Cai et al., 2015; Luo, Cai, Sedikides et al., 2014; Luo, Cai, \& Song, 2014; Luo et al., 2016; Luo, Shi et al., 2014; Zhou, Wong et al., 2018). As shown in Table 4, most phenotypes were influenced by genes and non-shared environments. In addition, these studies provided the genetic and environmental bases of the relationships between some related concepts. For example, the two dimensions of narcissism (i.e., intrapersonal grandiosity and interpersonal entitlement, agentic narcissism, and communal narcissism) were largely independent of each other in terms of their genetic and environmental sources (Luo, Cai, Sedikides et al., 2014; Luo, Cai, \& Song, 2014). However, some associations were driven by common genetic and non-shared environmental influences, such as the positive associations between name-liking and subjective wellbeing (Luo, Shi et al., 2014) and between global narcissism and impulsive buying (Cai et al., 2015). These findings have provided novel evidence for theories and deepen our understanding of society and personality psychology.

\section{Genetic and Environmental Influences on Cognitive and Other Factors}

We have examined the heritability of some phenotypes of cognitive factors and achieved important research results. For example, we found that the two components of BM perception have different genetic and environmental bases. The local BM was influenced mainly by genes; nevertheless, the global BM was influenced mainly by environment (Wang et al., 2018). This was also observed 
for the relationship between the two core cognitive features in schizophrenia, and spatial working memory had moderate heritability, but set-shifting was explained mostly by specific environments (Zhou, Li et al., 2018).

Furthermore, some studies have found that autistic traits and risk-taking were both influenced by genes and non-shared environments (Rao et al., 2018; Wang et al., 2018). The heritability rates for autistic traits and risk-taking were $60 \%(95 \%$ CI $[43,73])$ and $41 \%(95 \%$ CI $[26,53])$, respectively. These findings advance our understanding of the causes of individual differences in psychological traits, which is important in psychology and social sciences.

\section{Conclusions and Future Plans}

As a result of China's strict one-child policy, there are many Chinese families with one child, especially those in urban areas. Yet, we do not think that the representativeness of twin samples in the population will be influenced. First, there are also many families in China with more than one child. Based on an estimation and survey, by the end of 2015, the population of only children was 224.61 million and the population of children with siblings was 519.98 million (Li, Wang et al., 2018). The study results of twin samples can be generalized to this category of children with siblings. Second, the change of policy will lead to more families with more than one child. At the end of 2015, China decided to allow all couples to have two children, so there will be more children with siblings in China.

The BeTwiSt is an ongoing twin study that aims to unravel the interplay between genes and environment in the development of psychopathology. Some of the participants are now reaching adulthood; therefore, we are preparing to measure their emotional and behavioral problems, which are important outcome variables in adulthood (e.g., educational level and marital status), to increase our understanding of how experiences that occur during adolescence influence adult psychopathology. Moreover, we will expand our collaboration with other research groups to advance the development of behavioral genetics research.

Acknowledgments. We would like to thank the twins and their families, and schools participating in the BeTwiSt.

Financial support. This work was supported by the National Natural Science Foundation of China (grant number 31530031).

Conflict of interest. None.

\section{References}

Cai, H., \& Luo, Y. L. L. (2017). The heritability of implicit self-esteem: A twin study. Personality and Individual Differences, 119, 249-251.

Cai, H., Luo, Y. L. L., Shi, Y., Liu, Y., \& Yang, Z. (2016). Male = science, female = humanities. Social Psychological and Personality Science, 7, 412-419.

Cai, H., Shi, Y., Fang, X., \& Luo, Y. L. (2015). Narcissism predicts impulsive buying: phenotypic and genetic evidence. Frontiers in Psychology, 6, 881.

Chen, J., \& Li, X. (2013). Genetic and environmental influences on adolescent rumination and its association with depressive symptoms. Journal of Abnormal Child Psychology, 41, 1289-1298.

Chen, J., \& Li, X. (2014). Genetic and environmental etiologies of adolescent dysfunctional attitudes: A twin study. Twin Research and Human Genetics, $17,16-22$.

Chen, J., Li, X., Chen, Z., Yang, X., Zhang, J., Duan, Q., \& Ge, X. (2012). Optimization of zygosity determination by questionnaire and DNA genotyping in Chinese adolescent twins. Twin Research and Human Genetics, 13, 194-200.
Chen, J., Li, X., \& McGue, M. (2012). Interacting effect of BDNF Val66Met polymorphism and stressful life events on adolescent depression. Genes, Brain and Behavior, 11, 958-965.

Chen, J., Li, X., \& McGue, M. (2013). The interacting effect of the BDNF Val66Met polymorphism and stressful life events on adolescent depression is not an artifact of gene-environment correlation: Evidence from a longitudinal twin study. Journal of Child Psychology and Psychiatry, 54, 1066-1073.

Chen, J., Li, X., Natsuaki, M. N., Leve, L. D., \& Harold, G. T. (2014). Genetic and environmental influences on depressive symptoms in Chinese adolescents. Behavioral Genetics, 44, 36-44.

Chen, J., Li, X., Zhang, J., Natsuaki, M. N., Leve, L. D., Harold, G. T., . . Ge, X. (2012). The Beijing Twin Study (BeTwiSt): A longitudinal study of child and adolescent development. Twin Research and Human Genetics, 16, 91-97.

Chen, J., Yu, J., Li, X., \& Zhang, J. (2015). Genetic and environmental contributions to anxiety among Chinese children and adolescents - A multiinformant twin study. Journal of Child Psychology and Psychiatry, 56, 586-594.

Chen, J., Yu, J., Liu, Y., Zhang, L., \& Zhang, J. (2015). BDNF Val66Met, stress, and positive mothering: Differential susceptibility model of adolescent trait anxiety. Journal of Anxiety Disorders, 34, 68-75.

Chen, J., Yu, J., \& Zhang, J. (2016). Investigating unique environmental influences of parenting practices on youth anxiety: A monozygotic twin differences study. International Journal of Behavioral Development, 40, 205-212.

Chen, J., Yu, J., Zhang, J., Li, X., \& McGue, M. (2015). Investigating genetic and environmental contributions to adolescent externalizing behavior in a collectivistic culture: A multi-informant twin study. Psychological Medicine, 45, 1989-1997.

Chen, J., Yu, J., Zhang, L., Li, X., \& Zhang, J. (2014). Etiological heterogeneity of symptom dimensions of adolescent depression. PsyCh Journal, 3, 254-263.

Cui, Y., Liu, B., Zhou, Y., Fan, L., Li, J., Zhang, Y., .. Jiang, T. (2016). Genetic effects on fine-grained human cortical regionalization. Cerebral Cortex, 26, 3732-3743.

Hou, J., Chen, Z., Natsuaki, M. N., Li, X., Yang, X., Zhang, J., \& Zhang, J. (2013). A longitudinal investigation of the associations among parenting, deviant peer affiliation, and externalizing behaviors: A monozygotic twin differences design. Twin Research and Human Genetics, 16, 698-706.

Jansen, A. G., Mous, S. E., White, T., Posthuma, D., \& Polderman, T. J. (2015). What twin studies tell us about the heritability of brain development, morphology, and function: A review. Neuropsychology Review, 25, 27-46.

Krause, E. D., Vélez, C. E., Woo, R., Hoffmann, B., Freres, D. R., Abenavoli, R. M., \& Gillham, J. E. (2017). Rumination, depression, and gender in early adolescence: A Longitudinal study of a bidirectional model. The Journal of Early Adolescence, 38, 923-946.

Li, H., Wang, R., \& Ren, Y. (2018). Analysis of the number of only children and family structure since the one-child policy. Statistics and Decision-Making, $34,99-104$.

Li, M., Chen, J., Li, N., \& Li, X. (2014). A twin study of problematic internet use: its heritability and genetic association with effortful control. Twin Research and Human Genetics, 17, 279-287.

Li, Z., Huang, J., Xu, T., Wang, Y., Li, K., Zeng, Y. W., . . Chan, R. C. K. (2018). Neural mechanism and heritability of complex motor sequence and audiovisual integration: A healthy twin study. Human Brain Mapping, 39, 1438-1448.

Luo, Y. L. L., Cai, H., Sedikides, C., \& Song, H. (2014). Distinguishing communal narcissism from agentic narcissism: A behavior genetics analysis on the agency-communion model of narcissism. Journal of Research in Personality, 49, 52-58.

Luo, Y. L. L., Cai, H., \& Song, H. (2014). A behavioral genetic study of intrapersonal and interpersonal dimensions of narcissism. Plos One, 9, e93403.

Luo, Y. L. L., Liu, Y., Cai, H., Wildschut, T., \& Sedikides, C. (2016). Nostalgia and self-enhancement. Social Psychological and Personality Science, 7, 857-866.

Luo, Y. L. L., Shi, Y., Cai, H., Wu, M., \& Song, H. (2014). Liking for name predicts happiness: A behavioral genetic analysis. Personality and Individual Differences, 69, 156-161.

Menon, V. (2011). Large-scale brain networks and psychopathology: A unifying triple network model. Trends in Cognitive Sciences, 15, 483-506.

Neale, M. C. (2003). A finite mixture distribution model for data collected from twins. Twin Research and Human Genetics, 6, 235-239.

Park, H., \& Poo, M.-M. (2012). Neurotrophin regulation of neural circuit development and function. Nature Reviews Neuroscience, 14, 7. 
Polderman, T. J., Benyamin, B., de Leeuw, C. A., Sullivan, P. F., van Bochoven, A., Visscher, P. M., \& Posthuma, D. (2015). Meta-analysis of the heritability of human traits based on fifty years of twin studies. Nature Genetics, 47, 702-709.

Rao, L.-L., Zhou, Y., Zheng, D., Yang, L.-Q., \& Li, S. (2018). Genetic contribution to variation in risk taking: A functional MRI twin study of the balloon analogue risk task. Psychological Science, 29, 1679-1691.

Wang, Y., Wang, L., Xu, Q., Liu, D., Chen, L., Troje, N. F., ... Jiang, Y. (2018). Heritable aspects of biological motion perception and its covariation with autistic traits. Proceedings of the National Academy of Sciences of the United States of America, 115, 1937-1942.

Winkeljohn Black, S., \& Pössel, P. (2015). Integrating Beck's cognitive model and the response style theory in an adolescent sample. Journal of Youth and Adolescence, 44, 195-210.
Zhang, L., Li, Z., Chen, J., Li, X., Zhang, J., \& Belsky, J. (2016). The BDNF Val66Met polymorphism interacts with maternal parenting influencing adolescent depressive symptoms: Evidence of differential susceptibility model. Journal of Youth and Adolescence, 45, 471-483.

Zhou, H. Y., Li, Z., Xie, D. J., Xu, T., Cheung, E. E. F., Li, H., \& Chan, R. C. K. (2018). Heritability estimates of spatial working memory and set-shifting in a healthy Chinese twin sample: A preliminary study. PsyCh Journal, 7, 144-151.

Zhou, H. Y., Wong, K. K., Shi, L. J., Cui, X. L., Qian, Y., Jiang, W. Q., . . Chan, R. C. K. (2018). Suspiciousness in young minds: Convergent evidence from non-clinical, clinical and community twin samples. Schizophrenia Research, 199, 135-141.

Zhou, Y., Chen, J., Luo, Y., Zheng, D., Rao, L.-L., Li, X., ... Zuo, X.-N. (2016). Genetic overlap between in-scanner head motion and the default network connectivity. bioRxiv. doi:10.1101/087023\%JbioRxiv 